\title{
Theoretical Analysis on the Impact of Total Damping Ratio on the Power Output of an Electromagnetic Vibration Energy Harvester
}

\author{
Faruq Muhammad Foong ${ }^{1}$, Chung Ket Thein ${ }^{1}$ and Beng Lee Ooi ${ }^{2}$ \\ ${ }^{1}$ School of Engineering and Physical Sciences, Heriot Watt University Malaysia \\ 2Intel PSG, PG 14, Plot 6, Bayan Lepas Technoplex, Medan Bayan Lepas, 11900 Penang, Malaysia.
}

\begin{abstract}
Vibration energy harvesting has emerged as a promising source of sustainable energy to power small electronics. This study investigates the effect of total damping on the power output of an electromagnetic vibration energy harvester. Analytical results show that an increase in the effective mass of the harvester increases the mechanical damping but decreases the electromagnetic damping. The total damping of the harvester displayed an increasing trend with the effective mass when the electromagnetic damping is lower that the mechanical damping but changed into a decreasing trend when the electromagnetic damping becomes larger than the mechanical damping. Findings also suggest that there is an optimum proof mass to beam mass ratio where the harvester would produce maximum power in both cases of where a constant and varying optimum load resistance were considered.
\end{abstract}

\section{Introduction}

Advancement in wireless sensor network (WSN) technologies have increased research in energy harvesting to find a sustainable source of energy to power these devices. While sources such as heat, light and wind energy harvesting was an option, vibration energy harvesting became an instant research attraction due to its promising advantages [1-3].

There exists several methods to convert the mechanical vibrational energy into electrical power with the two most common methods being piezoelectric conversion and electromagnetic induction. Kim et al. [4] reported that piezoelectric harvesters perform better at small volumes whereas electromagnetic harvesters surpassed piezoelectric at larger volumes. The performance of an electromagnetic vibration energy harvester is strongly dependent on the velocity of the vibrating beam, which can be strongly related to the damping of the system.

Generally, an electromagnetic harvester consist of two main sources of damping, which are the mechanical damping and the electromagnetic damping [5]. For a cantilever-beam based harvester, the mechanical damping originates from the internal friction of the vibrating beam. Damping due to internal friction is highly material specific. Hence, different material will exhibit different damping capacities. In an earlier study, Lazan [6] proposed that the mechanical damping of a structure can be strongly related to its maximum vibrating stress and its fatigue limit stress. The study suggest an increase in mechanical damping when the stress of the structure increase, which is generally true.

Electromagnetic damping arises from the interaction between the eddy current in a vibrating coil and a magnetic field, producing a force that retards the vibrating motion [7]. Consequently, the total damping of an electromagnetic harvester is simply the sum of its mechanical and electromagnetic damping. Damping plays a complex role in influencing the power output of an electromagnetic energy harvester, and this study aims to investigate the effect of the total damping on the power output of the harvester through analytical means.

\section{Governing equations}

Figure 1 shows the mechanism of a typical cantilever beam-based electromagnetic vibration energy harvester under harmonic base excitation.

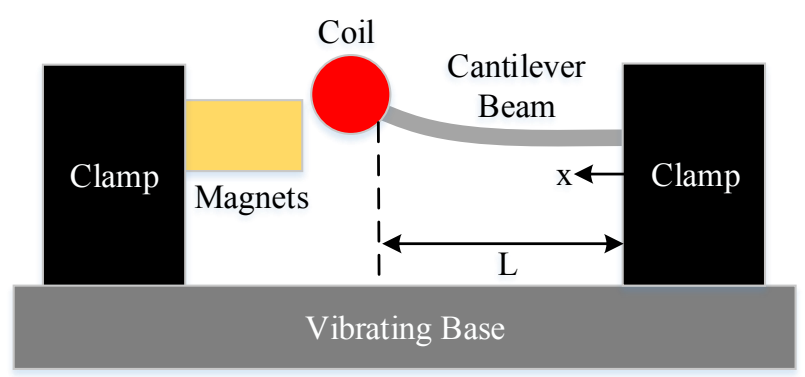

Figure 1. Schematic of a cantilever beam-based electromagnetic vibration energy harvester.

Based on Faraday's law of electromagnetic induction, power can be generated when the cantilever beam vibrates, causing the coil to cut through the magnetic field of the magnets. To fully model the effect of damping on the power output of the harvester, the governing equations relating the harvester's mechanical 
damping and electromagnetic damping to its power output must be considered simultaneously.

\subsection{Mechanical damping}

Assuming linear damping, the mechanical damping ratio of a vibrating structure can be related to its maximum stress by the following equation [6]

$$
\zeta_{m}=3619.5\left(\sigma_{m}{ }^{0.3} / \sigma_{f}^{-2.3}\right)+2887.1\left(\sigma_{m}{ }^{6} / \sigma_{f}^{-8}\right)
$$

where $\zeta_{m}$ is the mechanical damping ratio of the structure, $\sigma_{m}$ is the maximum stress experienced by the vibrating structure and $\sigma_{f}$ is the fatigue limit stress of the structure. Erturk and Inman [8] modelled the equation of motion of a clamp-free cantilever beam with proof mass under harmonic base excitation for first mode vibrations as the following

$$
z(X)=K(x) Y_{0} / 2 \zeta_{m}
$$

where $z(x)$ is the relative amplitude of the beam at position $x$ along the length of the beam, $K(x)$ is a constant derived from the inertial and static moment terms of the beam and its proof mass, $Y_{0}$ is the harmonic base excitation amplitude and $\zeta_{m}$ is the mechanical damping ratio of the beam. Here, the proof mass is assumed to be rigidly fixed onto the free-end of the beam. By applying the Euler-Bernoulli's bending theory, the maximum stress of the vibrating beam can be determine by equating $x=0$.

$$
\sigma_{m}=E h K^{\prime \prime}(0) Y_{0} / 4 \zeta_{m}
$$

where E is the Young's modulus of the beam, $h$ is the beam's thickness and $K^{\prime \prime}(0)$ is the second derivative of $K(x)$ with respect to $X$, evaluated at $X=0$. By substituting equation (3) into equation (1), the mechanical damping ratio of the beam can be determined analytically, provided that the material properties of the beam are known.

\subsection{Electromagnetic damping}

The electromagnetic damping of an electromagnetic vibration energy harvester is given by [9]

$$
\zeta_{e}=\left(N B L_{c}\right)^{2} / 2 m_{e} w_{n}\left(R_{c}+R_{L}\right)
$$

where $\mathrm{N}$ is the number of turns of coil, $B$ is the strength of the magnetic field, $L_{c}$ is the practical length of the coil, $m_{e}$ is the effective mass of the harvester, $w_{n}$ is the natural frequency of the harvester and $R_{c}$ and $R_{L}$ is the coil and load resistance. For a cantilever beam with a proof mass attached onto its free-end, the effective mass of the system can be approximated as [10]

$$
m_{e}=m_{p}+33 / 140 m_{b}
$$

where $m_{p}$ is the mass of the proof mass and $m_{b}$ is the mass of the cantilever beam.

\subsection{Power output}

Kulah and Najafi [11] reported that the maximum power output of an electromagnetic vibration energy harvester at the load resistance can be expressed as the following

$$
P_{\text {max }}=\left(N B L_{c} v\right)^{2} R_{L} /\left(R_{c}+R_{L}\right)^{2}
$$

where $P_{\max }$ is the maximum load power output of the harvester and $v$ is the peak velocity of the coil cutting though the magnetic field. Considering both mechanical and electromagnetic damping and assuming that the peak velocity is equal to the maximum velocity at the free-end of the cantilever beam where $x=L, v$ can be defined as

$$
V=w_{n} K(L) Y_{0} / 2\left(\zeta_{T}\right)
$$

where $\zeta_{T}$ is known as the total damping and is equal to the sum of the mechanical and the electromagnetic damping. It is easy to notice that the velocity term in equation (7) is actually a product of equation (2) and the beam's natural frequency.

\section{Results and discussion}

Equation (4) suggest that increasing the effective mass of the harvester would decrease the overall electromagnetic damping. However, this will was also lead to an increase in stress and mechanical damping as described by equation (1). The effect of effective mass on the total damping of an electromagnetic vibration energy harvester was investigated in this section. Here, two cases were considered with the first case assuming a constant optimum load resistance and the second case considering the change in optimum load resistance for every variation in effective mass. These two cases were investigated due to the different theory on optimum load resistance by previous authors.

\subsection{Case 1 - Constant optimum load resistance}

Yang et al. [12] reported that the optimum load resistance of an electromagnetic vibration energy harvester is equal to the coil resistance and unaffected by other parameters. Therefore, increasing the effective mass of the harvester would not change the optimum load resistance value. Table 1 states the parameters used in the analysis.

Table 1. Parameters used in the theoretical analysis.

\begin{tabular}{|c|c|}
\hline$E(\mathrm{GPa})$ & 69 \\
\hline$N$ & 400 \\
\hline$L_{c}(\mathrm{~mm})$ & 4.0 \\
\hline$R_{c}(\Omega)$ & 6.7 \\
\hline$\sigma_{f}(\mathrm{MPa})$ & 95.6 \\
\hline
\end{tabular}

The material properties of an aluminium beam was considered in Table 1 as equation (1) is only valid for metals [6]. A constant base excitation acceleration of $0.1 \mathrm{~g}$ was applied. Figure 2 describes the variation in electromagnetic and mechanical damping with effective 
mass for different magnetic field strengths, $B$. Figure 3 describes the corresponding total damping from Figure 2. Here, the effective mass term was translated into the mass ratio, $m_{r}$, of the proof mass to the beam mass as this will give a more general insight on the analysis.

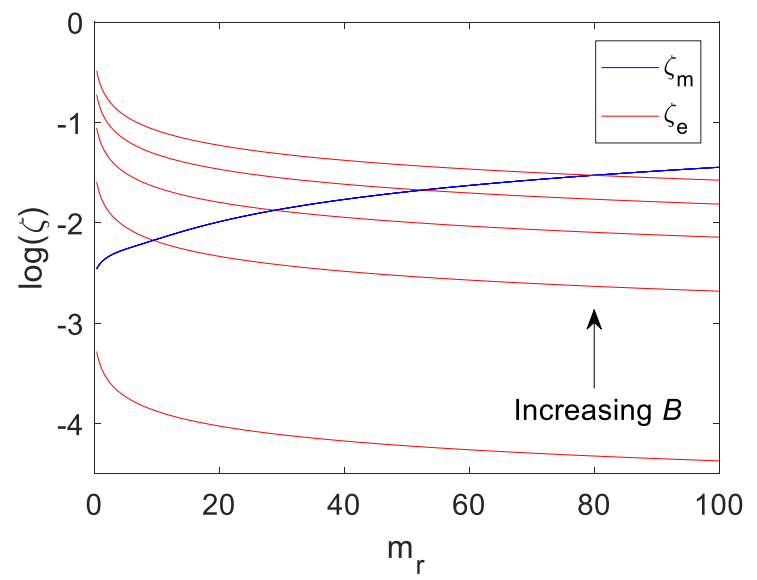

Figure 2. Case 1 variation in mechanical and electromagnetic damping ratio with mass ratio.

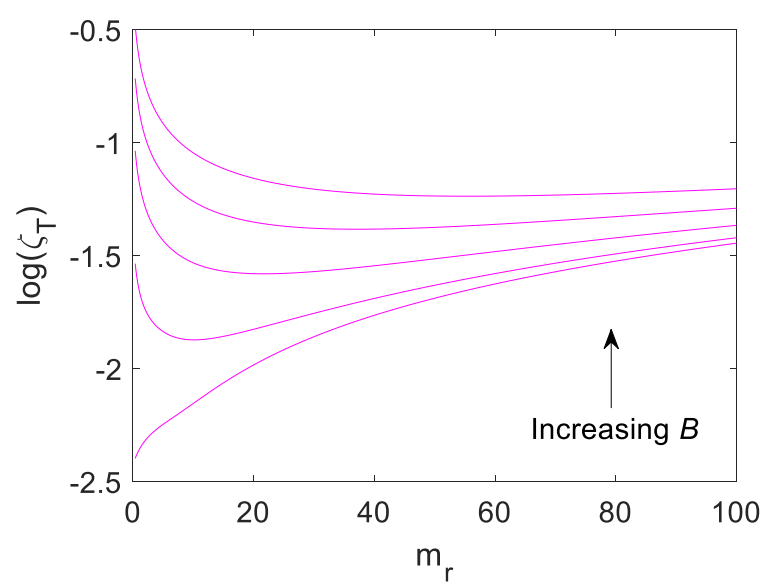

Figure 3. Case 1 variation in total damping ratio with mass ratio.

Figure 2 shows that increasing the mass ratio of the harvester results in an increase in mechanical damping and a decrease in electromagnetic damping. The results in Figure 3 illustrates that at regions where the electromagnetic damping is higher than the mechanical damping, the total damping decreases when the mass ratio increases. However, an opposite trend is seen when the electromagnetic damping is smaller than the mechanical damping. Overall, this analysis suggest that increasing the mass ratio of the electromagnetic harvester is favourable when the electromagnetic damping is larger than the mechanical damping, as this will decrease the total damping and hence an increase in gain. However, equation (6) suggest that a decrease in total damping does not necessarily lead to an increase in power as there are other parameters that must be considered such as the natural frequency on the harvester. Increasing the mass ratio would also result in a decrease in the natural frequency. Therefore, the output power was plotted against the variation in mass ratio to investigate this matter. Figure 4 shows the variation in the maximum load power for each different mass ratio input. Each curve in Figure 4 corresponds to the results in Figures 2 and 3.

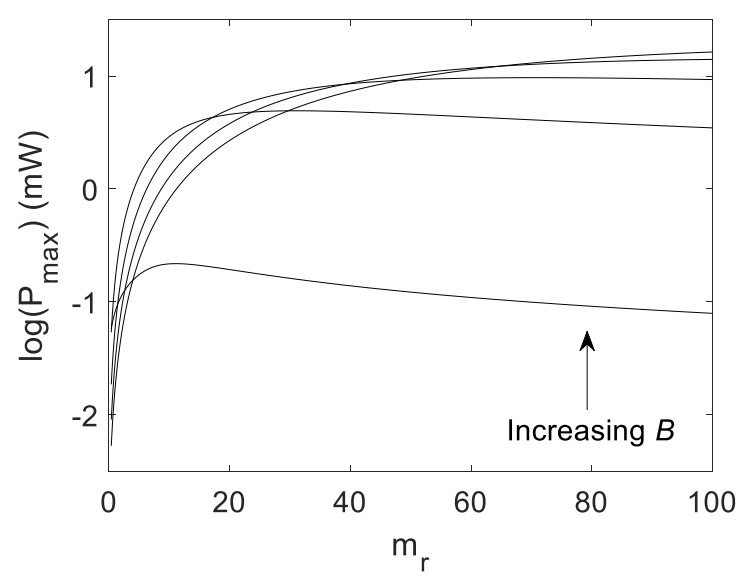

Figure 4. Case 1 variation in maximum load power output with mass ratio.

Figure 4 shows that an optimum mass ratio exist where the harvester would generate a maximum load power output. This optimum value increases when the magnetic field strength increases. This analysis also suggest that for every different harvester designs, the harvester has an optimum operational natural frequency. However, for cases of large optimum mass ratios, practicality may become an issue.

\subsection{Case 2 - Varying optimum load resistance}

Saha et al. [13] stated that when the electromagnetic damping of an electromagnetic vibration harvester becomes significant, the optimum load resistance does not equal to the coil resistance. The analysis in Case 1 was repeated by considering the change in optimum load resistance for every variation of effective mass.

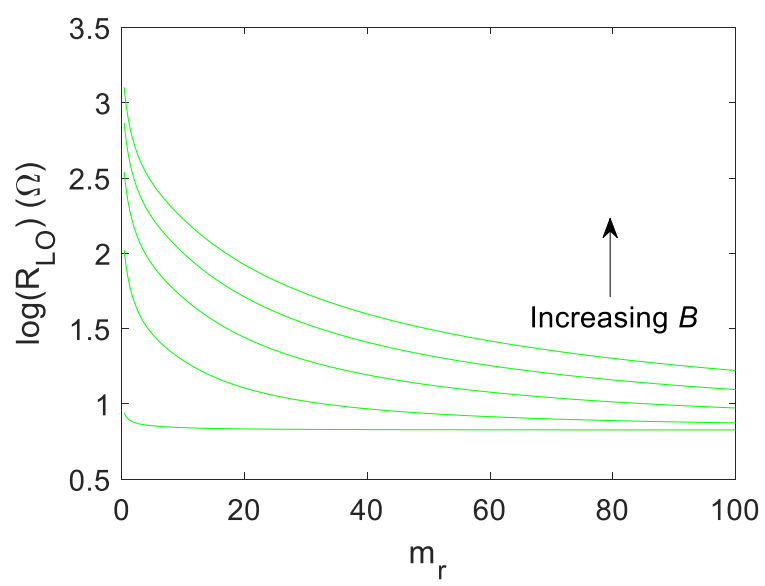

Figure 5. Variation in optimum load resistance with mass ratio

All analysis in Case 2 uses the same parameters applied as in Case 1. Figure 5 describes the variation in optimum load resistance and mass ratio. The optimum load resistance was determine using equation (6), in where the optimum value was selected based on the load 
resistance that resulted in the maximum load power output. It is shown that increasing the mass ratio of the harvester decrease the optimum load resistance value. Figure 6 shows the variation in mechanical damping and electromagnetic damping for Case 2 and Figure 7 describes the corresponding total damping.

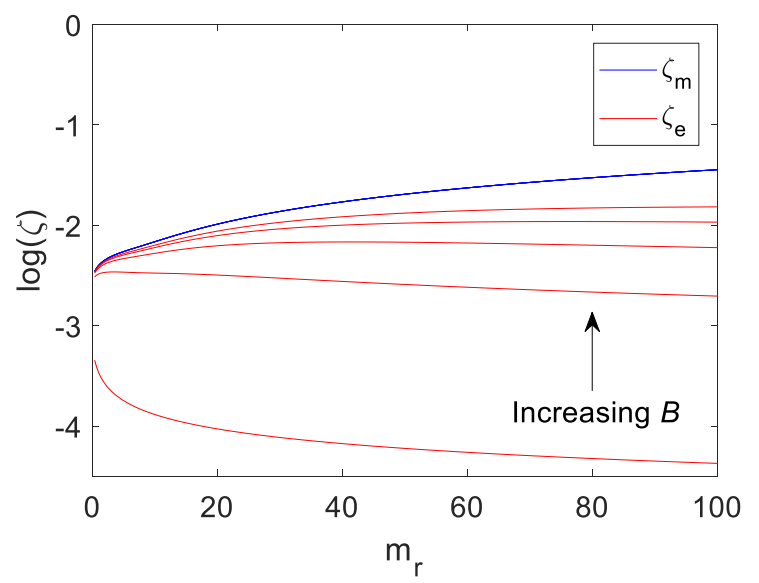

Figure 6. Case 2 variation in mechanical and electromagnetic damping ratio with mass ratio.

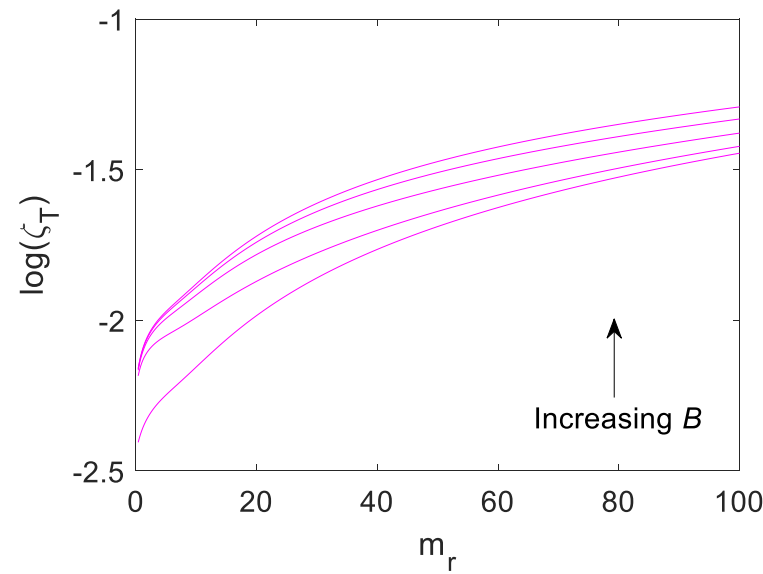

Figure 7. Case 2 variation in total damping ratio with mass ratio.

Analytical results in Figures 6 and 7 portrays a considerably different result than Case 1. It is shown that when the variation in optimum load resistance for every different mass ratio is considered, the electromagnetic damping will always be lower than the mechanical damping for any mass ratios, even under high magnetic field strength. This resulted in an increasing trend in the total damping for all magnetic strength variation as seen in Figure 7. Nevertheless, the power output results for Case 2 is somewhat similar to Case 1. Figure 8 shows that there also exist an optimum mass ratio or natural frequency in where the harvester would generate maximum load power. It worth to note that if the mechanical damping was assumed to be constant for all mass ratios, then the optimum mass ratio shown in Figures 4 and 8 would not exist, as the load power output would display a continuously increasing trend with when the mass ratio increases. However, this assumption is an over-generalisation for cantilever beam-based harvesters and may lead to large errors when performing large-scale optimisation.

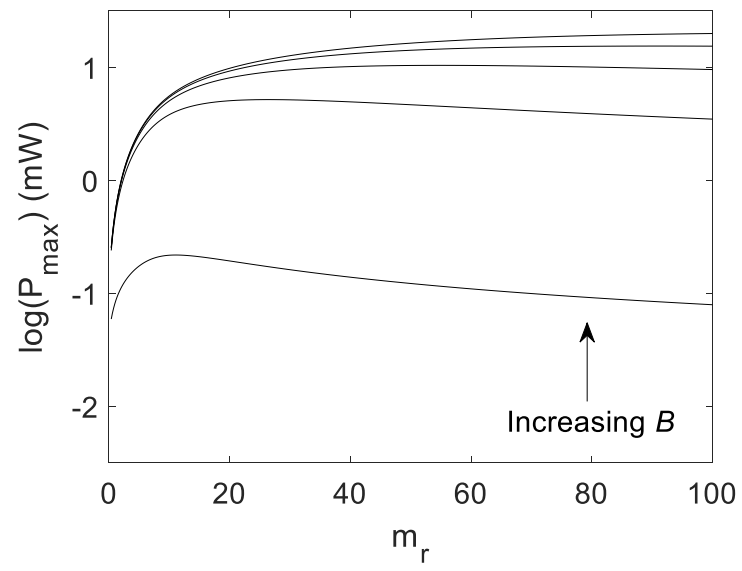

Figure 8. Case 2 variation in maximum load power output with mass ratio.

\section{Conclusion}

This study presents a theoretical analysis on the influence of total damping on the power output of an electromagnetic vibration energy harvester. Initially, the governing equations for the equation of motion and the power output of a cantilever beam-based electromagnetic vibration energy harvester were derived. Both variations in mechanical damping and electromagnetic damping for different harvester specifications were considered based on models developed from past literatures. Analytical results shown that increasing the effective mass of a cantilever beam-based harvester would result in an increase in the mechanical damping and a decrease in the electromagnetic damping. The total damping of the harvester displayed an increasing trend with effective mass at regions where the electromagnetic damping is lower than the mechanical damping. An opposite trend was recorded when the electromagnetic damping is larger. If the change in optimum load resistance was considered for every different effective mass variation, the electromagnetic damping will always fall below the mechanical damping. In terms of power output, results described that there is an optimum mass ratio value where the harvester would produce maximum power. This optimum value varies according to the harvester's design specifications. Hence, this also suggest that different harvester designs would have a different optimum operational natural frequency. Future works include deriving the exact expression for the condition of optimum mass ratio and verifying the results through experimental methods. The analytical analysis presented in this work are only valid for electromagnetic vibration energy harvesters, and exploration into damping analysis for other types of vibration energy harvesters may be considered in the future.

\section{Acknowledgement}

The authors would like to thank the Fundamental Research Grant Scheme (FRGS) from Ministry of Higher Education (MOHE) Malaysia, Grant No: 
FRGS/1/2016/TK03/HWUM/03/1, for funding this research.

\section{References}

[1] S. Dua,, Y. Jia, S. Chena, C. Zhaoa, B. Sunc, E. Arroyoa, A.A. Seshiaa, Sens. Actuat. A, 263 (2017)

[2] C.K. Thein, J.S. Liu, Eng. Comput., 33 (2017)

[3] C. Wei, X. Jing, Renew. Sustain Energy Rev., 74 (2017)

[4] S.G. Kim, S. Priya, I. Kanno, MRS Bulletin, 37.11 (2012)

[5] Y. Tan, Y. Dong, X. Wang, J. Microelectromech., 26(1) (2017)

[6] B.J. Lazan, Damping of materials and members in structural mechanics (Pergamon Press, London, 1968)
[7] E. Arroyo, A. Badel, Sens. Actuat. A, 171 (2011)

[8] A. Erturk, D.J. Inman, J. Intel. Mater. Syst. Struc., 19 (2008)

[9] B.L. Ooi, J.M. Gilbert, Sens. Actuat. A, 213 (2014)

[10] H. Yu, J. Zhou, L. Deng, Z. Wen, Sens., 14 (2014)

[11] H. Kulah, K. Najafi, IEEE Sens. J., 8(3) (2008)

[12] B. Yang, C. Lee1, W. Xiang, J. Xie1, J. Han He, R.K. Kotlanka, S.P. Low, H. Feng, J. Micromech. Microeng., 19 (2009)

[13] C.T. Saha, T. O’Donell, H. Loder, S. Beeby and J. Tudor, IEEE Trans. Magnetics, 42(10) (2006) 